\title{
Utilidad de la endoscopia intraoperativa en el bypass gástrico en Y de Roux laparoscópico
}

\section{Utility of intraoperative endoscopy in laroscopic Roux-Y gastric bypass}

\author{
Martha J. Santacreo-Posas ${ }^{1 *}$, Martín Antonio-Manrique ${ }^{1}$, Miguel Á. Chávez-García ${ }^{1}$, Jony Cerna-Cardona ${ }^{1}$, \\ Giuseppe Briceño-Sáenz ${ }^{2}$, Lizeth S. Godínez-Franco ${ }^{1}$ y Magdalena B. Gómez-Cruz ${ }^{1}$ \\ ${ }^{1}$ Departamento de Cirugía; ${ }^{2}$ Departamento de Gastroenterología. Servicio de Endoscopia Gastrointestinal, Hospital Juárez de México, Ciudad de \\ México, México
}

\begin{abstract}
Resumen
Introducción: El bypass gástrico en Y de Roux laparoscópico (BGYRL) es el estándar de oro en cirugía bariátrica, sin embargo, su morbimortalidad es reconocida. Se han desarrollado técnicas para detectar complicaciones potencialmente prevenibles. El objetivo de este estudio es determinar la utilidad de la EIO en los pacientes sometidos a BGYRL. Material y métodos: Se incluyeron a pacientes sometidos a BGYRL en el Hospital Juárez de México en el periodo octubre 2016-febrero 2018. Resultados: Del total de 41 pacientes, 28 fueron sometidos a BGYRL con EIO y 13 a BGYRL sin EIO. El grupo de pacientes sometidos a EIO presentó una tasa más baja de fuga anastomótica postoperatoria (0 vs $7.69 \%$ ). La estancia hospitalaria fue más corta para los pacientes sometidos a EIO (4.32 vs 5.61 dias). Se obtuvo fuga de aire positiva con una tasa de $3.5 \%$ $(n=1)$. Conclusión: La EIO demostró beneficio en la detección y corrección de fuga anastomótica durante el BGYRL y redujo el tiempo de inicio de la vía oral resultando en una estancia hospitalaria más corta.
\end{abstract}

Palabras clave: Endoscopia intraoperatoria. Cirugía bariátrica. BGYRL. Endoscopia intraoperatoria. Fuga de anastomosis.

\begin{abstract}
Background: Laparoscopic Roux-en-Y Gastric Bypass (LGBYB) is the gold standard in bariatric surgery and involves a recognized morbidity and mortality. Several techniques have been developed to detect potentially preventable complications. The objective of this study is to determine the usefulness of Intraoperative Endoscopy (EIO) in patients undergoing LYRGB. Material and methods: Patients undergoing LGBT at the Hospital Juárez de México in the period from October 2016 to February 2018 were included. Results: Of a total of 41 patients, 28 underwent LYGB with IOE and 13 underwent LYGB without IOE. The postoperative anastomotic leak rate was lower in the group of patients undergoing IOS (0 vs $7.69 \%)$. The hospital stay was shorter for patients undergoing IOS (4.32 vs 5.61 days). Positive air leakage was obtained with a rate of $3.5 \%$. Conclusion: IOS demonstrated benefit in the detection and correction of anastomotic leak during LYGBY and reduced the start time of the oral route, resulting in a shorter hospital stay.
\end{abstract}

Key words: Intraoperative endoscopy. Bariatric surgery. LRYGB. Leak. Stricture.

Correspondencia:

*Martha J. Santacreo-Posas

E-mail: marthasantacreo@gmail.com licencia CC BY-NC-ND (http://creativecommons.org/licenses/by-nc-nd/4.0/).

Disponible en internet: 09-04-2021

Rev Hosp Jua Mex. 2021;88(1):2-7

www.revistahospitaljuarez.com 


\section{Introducción}

Desde que Wittgrove, en 1993, introdujo el bypass gástrico en $\mathrm{Y}$ de Roux vía laparoscópica (BGYRL) y dio seguimiento a 500 pacientes de 700 operados con buenos resultados a largo plazo, este se ha convertido en una alternativa ampliamente aceptada con ventajas significativas, resultando en una marcada disminución del malestar perioperatorio, reducción de la estancia hospitalaria y los costos hospitalarios, y un retorno más temprano a un estilo de vida completo y productivo'.

Actualmente el BGYRL es el estándar de oro en el tratamiento quirúrgico de la obesidad ${ }^{2,3}$. Sin embargo, este conlleva una morbimortalidad reconocida secundaria a complicaciones como la fuga anastomótica de la gastroyeyunoanastomosis (GYA), principal causa de morbimortalidad e incremento en los costos de atención ${ }^{4-8}$. Se han desarrollado nuevas técnicas para detectar oportunamente esta y otras complicaciones. Dentro de estas técnicas, la endoscopia intraoperatoria (EIO) ha demostrado ser costo-efectiva en disminuir sustancialmente la morbilidad, detectando complicaciones intraoperatorias y su resolución². Este estudio tiene como objetivo determinar la utilidad de la EIO en la detección de fugas anastomóticas en los pacientes sometidos a BGYRL. El objetivo secundario fue identificar otras complicaciones intraoperatorias del BGYRL potencialmente prevenibles.

\section{Metodología}

\section{Diseño del estudio}

Estudio transversal, retrospectivo, descriptivo y unicéntrico. Se analizaron los siguientes parámetros en los pacientes: media de edad, número de pacientes que presentaron fugas en el intraoperatorio y el postoperatorio, el adecuado paso del endoscopio a través de la GYA, días de estancia hospitalaria, tiempo quirúrgico, tipo de sutura utilizada para la construcción de la GYA, número de pacientes con estenosis anastomótica y su tratamiento endoscópico.

\section{Población de estudio}

Todos los pacientes sometidos a BGYRL en el Hospital Juárez de México en el periodo de octubre de 2016-febrero de 2018.

\section{Criterios de inclusión}

Pacientes con obesidad mórbida sometidos a BGYRL.

\section{Criterios de exclusión}

Menores de 18 años, tratamiento quirúrgico de la obesidad diferente al bypass laparoscópico (manga gástrica, banda gástrica), cirugía no por vía laparoscópica en el tratamiento de la obesidad, información incompleta en el expediente clínico.

\section{Tamaño mínimo de muestra}

Se estudiaron todos los pacientes sometidos a bypass gástrico laparoscópico en el periodo determinado.

\section{Resultados}

Solo 41 pacientes cumplieron con los criterios de inclusión para esta revisión, 28 (68.3\%) fueron sometidos a BGYRL con EIO y $13(31.7 \%)$ a BGYRL sin EIO. Sexo: 34 mujeres $(82.9 \%)$ y 7 varones $(17.1 \%)$; con media de edad de 39.73 años (rango: 25-59 años). La media preoperatoria de índice de masa corporal (IMC) fue de $42.8 \mathrm{~kg} / \mathrm{m}^{2}$ (rango: $32-54.03 \mathrm{~kg} / \mathrm{m}^{2}$ ). En 29 (70.7\%) obesidad grado III, 9 (22\%) con obesidad grado II y $3(7.3 \%)$ con obesidad grado I (Fig. 1).

En relación con el riesgo anestésico, 27 (65.9\%) fueron clasificados como grado III y 14 (34.1\%) como grado II, de acuerdo con la clasificación de la Sociedad Americana de Anestesiología (ASA). El riesgo cardiovascular fue evaluado de acuerdo con la clasificación de Goldman; 35 pacientes (85.4\%) fueron clasificados como Goldman I y 6 (14.6\%) como Goldman II (Tabla 1). El $78 \%$ de los pacientes tenían una o más comorbilidades, la diabetes mellitus tipo 2 (DM2), con un $51.6 \%$, y la hipertensión arterial sistémica (HAS), con un $46.3 \%$, fueron las más frecuentes, seguidas de la dislipidemia, con el $29.3 \%$. Otras comorbilidades fueron hernia hiatal $(7.3 \%)$, resistencia a la insulina (7.3\%), apnea del sueño $(4.9 \%)$, hiperuricemia $(4.9 \%)$, hipotiroidismo $(4.9 \%)$, enfermedad por reflujo gastroesofágico (2.4\%) y endometriosis (2.4\%) (Fig. 1).

El tiempo quirúrgico fue en promedio de 172 minutos (rango: 140-270 minutos) para los pacientes con BGYRL con EIO y de 171 minutos (rango: 105380 minutos) para los pacientes con BGYRL sin EIO $(p=0.03318)$. Dos pacientes tuvieron tiempo quirúrgico más prolongado de lo esperado, uno de ellos 320 minutos debido a múltiples adherencias retrogástricas y el otro 380 minutos debido a prueba neumática positiva, realizada con sonda nasogástrica y reconstrucción de la GYA. La estancia hospitalaria promedio fue 


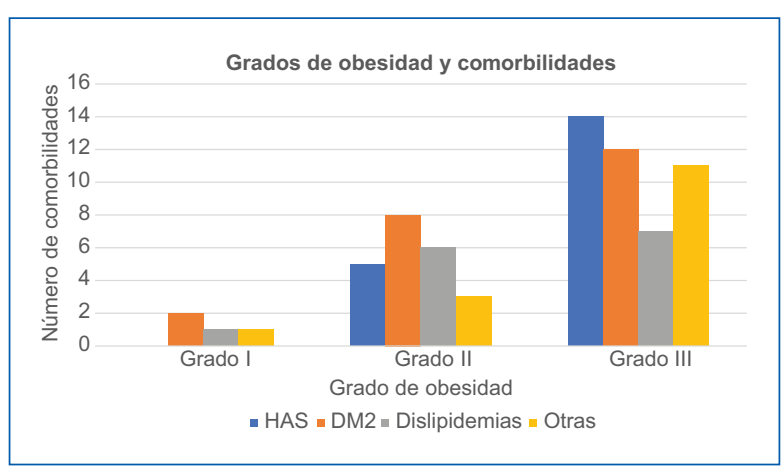

Figura 1. Relación entre grado de obesidad y comorbilidades. HAS: hipertensión arterial sistémica; DM2: diabetes mellitus tipo 2.

significativamente más corta para los pacientes sometidos a EIO (4.32 vs. 5.61 días; $p=0.037481$ ); la mayoría de los pacientes no sometidos a EIO $77 \%(n=10)$ permanecieron más de 72 horas en ayuno, hasta demostrar con estudio de imagen con material hidrosoluble ausencia de fuga a cualquier nivel (Fig. 2).

No hubo diferencia estadísticamente significativa en cuanto a la presentación de estenosis anastomótica. A todos los pacientes del grupo de BGYRL con EIO se les realizó prueba neumática en el transoperatorio al momento de hacer la endoscopia (Fig. 3).

La EIO detectó prueba de fuga positiva en la GYA en $1 / 28$ paciente (3.5\%), al que se le realizó reforzamiento de la línea de sutura. De los 27 pacientes con EIO con prueba de fuga negativa, ninguno presentó datos clínicos sugestivos de fuga anastomótica en el postoperatorio y fueron dados de alta sin eventualidades. A pesar de que los pacientes sometidos a EIO presentaron una tasa más baja de fuga anastomótica en el postoperatorio, el grado de significación (p) no fue estadísticamente significativo (0 vs. $7.69 \% ; p=0.3171$ ) (Tabla 2). Se realizó sutura manual en el $82 \%$ (23/28) de las GYA; la sutura de la anastomosis yeyunoyeyunal, del reservorio gástrico y del estómago excluido se realizaron con sutura mecánica con grapadora lineal en todos los pacientes; se completaron todas las EIO sin presentar complicaciones durante o después del procedimiento.

\section{Discusión}

La obesidad se ha convertido en uno de los problemas mundiales de salud más importantes, sobre todo por las comorbilidades asociadas que resultan en una mala calidad de vida de las personas afectadas y en el incremento de los costos en salud, surgiendo la
Tabla 1. Características de los pacientes

\begin{tabular}{l|c|}
\hline Sexo (relación H: M) & $1: 4.8$ \\
\hline Edad (años) & $39.7(25-59)$ \\
\hline IMC kg/m² & $42.8(32-54)$ \\
\hline Clasificación de ASA & $3(65.9 \%)$ \\
\hline Clasificación de Goldman & $1(85.4 \%)$ \\
\hline $\begin{array}{l}\text { H: hombre; M: mujer; IMC: índice de masa corporal; ASA: Sociedad Americana de } \\
\text { Anestesiología. }\end{array}$
\end{tabular}

Tabla 2. Complicaciones postoperatorias

\begin{tabular}{|l|c|c|c|}
\hline & Con EIO & Sin EIO & Valor de p \\
\hline Fuga GYA & $0 \%(n=0 / 28)$ & $7.69 \%(n=1 / 13)$ & 0.3171 \\
\hline $\begin{array}{l}\text { Estenosis } \\
\text { GYA }\end{array}$ & $7.69 \%(n=1 / 13)$ & $7.69 \%(n=1 / 13)$ & 0.539 \\
\hline
\end{tabular}

GYA: gastroyeyunoanastomosis; EIO: endoscopia intraoperatoria.

necesidad de tratamientos efectivos para combatir esta patología. La cirugía bariátrica ha resultado más efectiva que los tratamientos no quirúrgicos para la obesidad. Debido a sus buenos resultados, el BGYR es de los procedimientos bariátricos más utilizados en el mundo. Puede abordarse mediante laparoscopia o cirugía abierta y consiste en crear una pequeña bolsa a partir del estómago proximal (reservorio gástrico), excluyendo el resto del estómago y el duodeno del trayecto de los alimentos (rama biliopancreática). Una extremidad yeyunal se eleva y se anastomosa al reservorio gástrico (GYA) creando la rama de Roux, que tiene un componente corto de terminación ciega y una rama «alimenticia» de flujo anterógrado de 80 a $120 \mathrm{~cm}$ de longitud, que conduce a la anastomosis yeyunoyeyunal en su porción más distal ${ }^{9}$ (Fig. 4).

El BGYR con abordaje laparoscópico se considera el estándar de oro para el tratamiento de la obesidad, reemplazando por completo al abordaje abierto y ofreciendo múltiples ventajas como la reducción del dolor incisional y una menor tasa de complicaciones pulmonares, con resultados similares en cuanto a pérdida de peso y resolución de comorbilidades médicas asociadas $^{10,11}$. Sin embargo, estos beneficios de la cirugía bariátrica laparoscópica pueden perderse si las tasas de complicaciones son muy altas y los pacientes no son manejados adecuadamente ${ }^{11}$. 


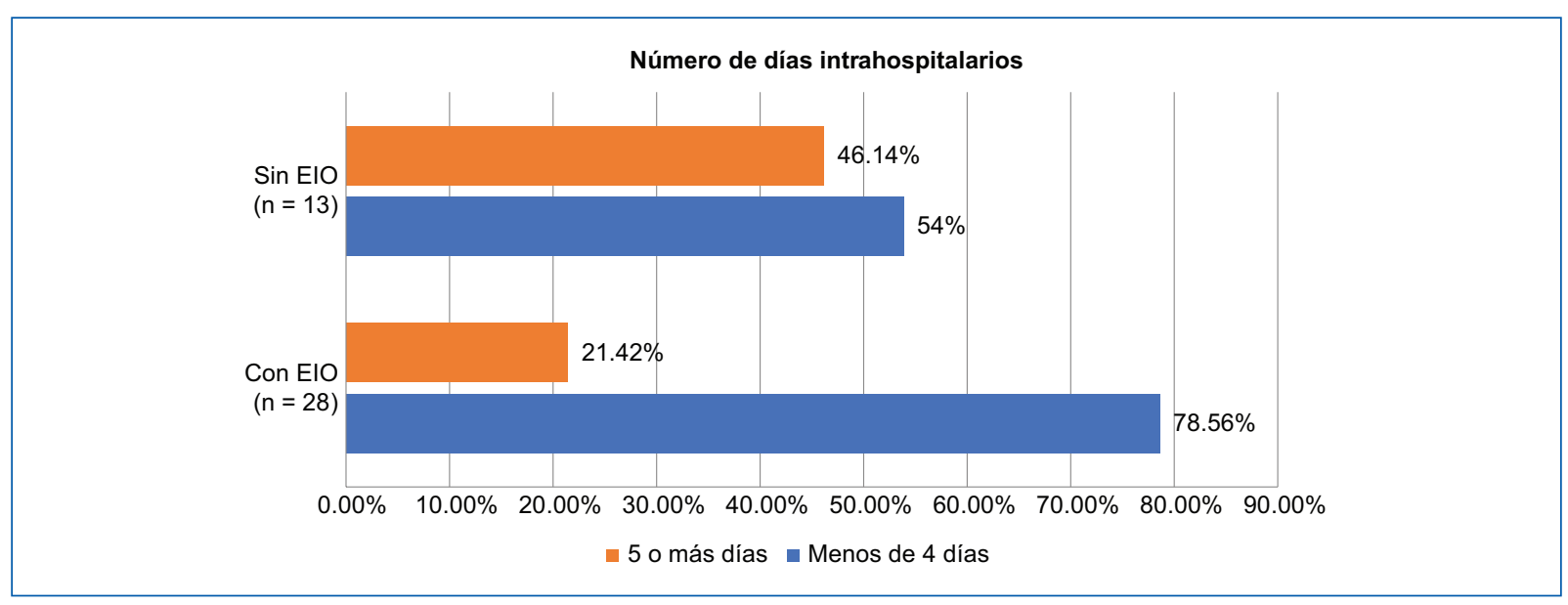

Figura 2. Tiempo de estancia hospitalaria.

EIO: endoscopia intraoperatoria.

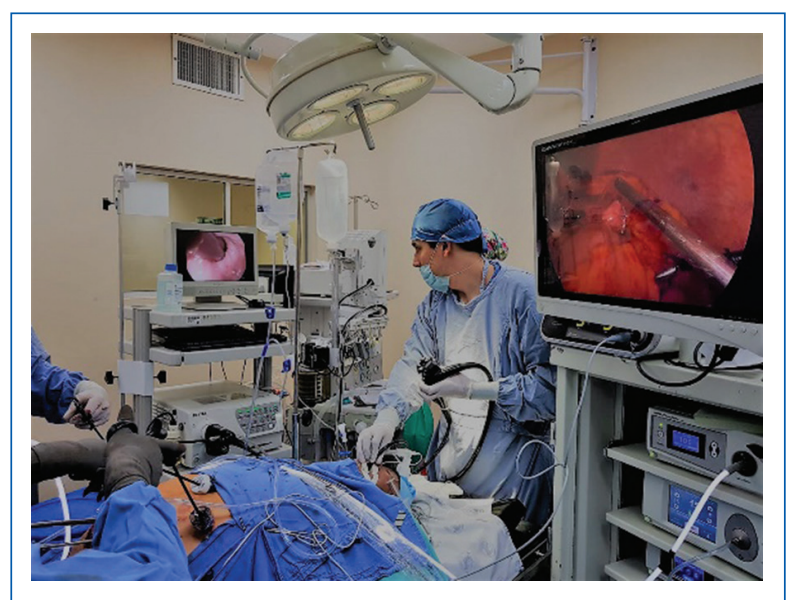

Figura 3. Bypass gástrico, prueba neumática endoscopia intraoperatoria, Hospital Juárez de México.

Al Hadad, et al. (2015) estudiaron 282 pacientes sometidos a BGYRL a los que se les realizó EIO ordinaria, siendo el $76 \%$ del sexo femenino, con un IMC promedio de $48 \mathrm{~kg} / \mathrm{m}^{2}$. En nuestra serie, similar a los otros estudios, predominó el sexo femenino, representado por el $82.9 \%$. Sin embargo, nuestro promedio de IMC $\left(42.8 \mathrm{~kg} / \mathrm{m}^{2}\right)$ resultó más bajo que el reportado en otros centros $\mathrm{s}^{2,3,10}$. La mayor parte de nuestros pacientes $(78 \%)$ presentaron dos o más comorbilidades, la DM2 fue la de mayor frecuencia (51.6\%), seguida de la HAS $(46.3 \%)$ y la dislipidemia $(29.3 \%)$, resultados similares a otro estudio mexicano, de ValenzuelaSalazar, et al. (2017), con la HAS en primer lugar seguida de dislipidemia y DM2 (60, 42 y $40 \%$, respectivamente) ${ }^{12}$. El tiempo operatorio en promedio fue de 172 minutos para los pacientes con BGYRL con EIO y de 171 minutos para aquellos sin EIO; esta diferencia no es estadísticamente significativa debido a que en dos de los pacientes a los que no se les realizó EIO se reportó un tiempo operatorio mayor a 300 minutos y la muestra de este grupo era menos de la mitad del grupo que fue sometido a EIO (13 y 28 respectivamente). La literatura reporta un mayor número de minutos de cirugía (194 minutos) cuando se realiza una $\mathrm{EIO}^{12}$.

Haddad, et al. (2012) analizaron el rol de la EIO en 2,311 pacientes sometidos a BGYRL y detectaron fugas intraoperatorias en el $3.5 \%$ de su muestra, y Alasfar et al. (2010) reportan en un estudio con 122 pacientes, una tasa de detección de fugas del $3.7 \%$ utilizando la $\mathrm{EIO}^{2,5}$.

En nuestro estudio la EIO detectó prueba de fuga positiva en la GYA en 1 de los 28 pacientes (3.5\%), al que se le realizó reforzamiento de la línea de sutura y no presentó datos clínicos de fuga en el postoperatorio. Esto nos lleva a que la detección temprana de la fuga anastomótica disminuye las consecuencias inherentes a esta complicación ${ }^{3}$.

Ramanathan, et al. demostraron en el año 2000 un $10 \%$ de incidencia de fugas de aire intraoperatorias en 182 pacientes sometidos a BGYRL y un $3.8 \%$ de incidencia para fugas postoperatorias después de la reparación durante el procedimiento inicial. Es de relevancia señalar que de los 27 pacientes con EIO con prueba de fuga negativa, ninguno presentó datos clínicos de fuga anastomótica en el postoperatorio, lo 


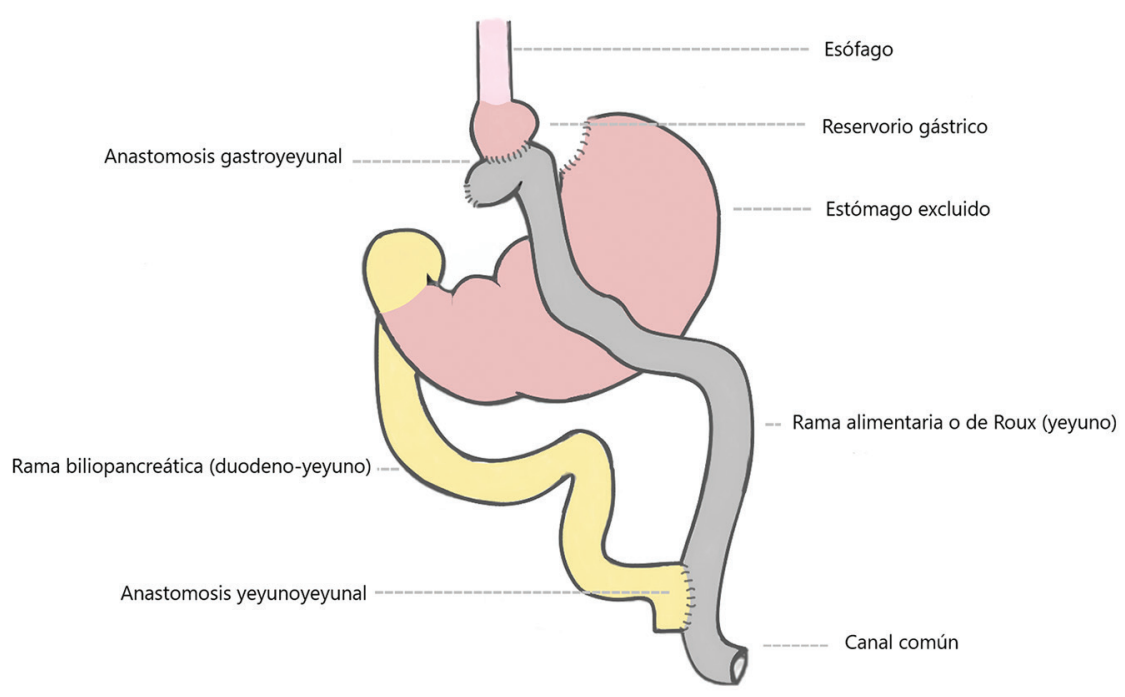

Figura 4. Diagrama de la reconstrucción anatómica en el bypass gástrico en $Y$ de Roux.

que traduce un VPN del $100 \%$, muy similar al de Al Hadad, et al. (2015), que fue del $99.5 \%$, y es en este punto donde destaca la utilidad de la EIO en el BGYRL. En nuestra serie el promedio de estancia hospitalaria fue de forma estadísticamente significativa más corta en los pacientes sometidos a EIO (4.32 vs. 5.61 días; $p=0.037481$ ). Estos resultados son similares a los de otros estudios $(2.44 \pm 1.6$ vs. 3.46 $\pm 2.7)^{3,12}$.

Nuestro estudio tiene varias limitaciones. Es una revisión retrospectiva de un estudio de base de datos de un solo centro. Debido al corto tiempo que tiene el tratamiento quirúrgico de la obesidad en nuestro hospital, el tamaño de la muestra es pequeño y, agregado a esto, es heterogénea. La endoscopia depende en gran medida del operador y la experiencia debe estar disponible dentro del equipo quirúrgico para permitir la flexibilidad en la realización de la EIO, y el endoscopista que la realiza debe estar familiarizado con la anatomía del BGYRL.

\section{Conclusiones}

- La EIO demostró beneficios en cuanto a la detección y corrección de fugas anastomóticas intraoperatorias, sin embargo, en nuestro estudio los pacientes sometidos a BGYRL tuvieron la misma probabilidad de presentar complicaciones.

- La EIO con prueba de fuga negativa reduce de manera significativa la estancia hospitalaria, el costo de atención y el inicio de la vía oral; no es necesario realizar otras pruebas para la detección de fuga en el postoperatorio.

- Las limitantes de nuestro estudio fueron el tamaño y la heterogeneidad de la muestra, además del corto tiempo que tiene el tratamiento quirúrgico de la obesidad en nuestro hospital, sin embargo, consideramos que se debe continuar la investigación y en un futuro determinar el impacto de la EIO con el objetivo de disminuir la incidencia de fuga anastomótica en el tratamiento quirúrgico de la obesidad.

\section{Agradecimientos}

Agradecemos a todos los médicos adscritos, residentes y revisores de la revista del Hospital Juárez de México que participaron e hicieron posible la realización de este estudio.

\section{Financiamiento}

Los autores no recibieron patrocinio para llevar a cabo este artículo.

\section{Conflicto de intereses}

Los autores declaran no tener conflicto de intereses alguno. 


\section{Responsabilidades éticas}

Protección de personas y animales. Los autores declaran que para esta investigación no se han realizado experimentos en seres humanos ni en animales.

Confidencialidad de los datos. Los autores declaran que han seguido los protocolos de su centro de trabajo sobre la publicación de datos de pacientes.

Derecho a la privacidad y consentimiento informado. Los autores han obtenido el consentimiento informado de los pacientes y/o sujetos referidos en el artículo.

\section{Bibliografía}

1. Wittgrove AC, Clark GW. Laparoscopic gastric bypass, Roux-en-Y 500 patients: technique and results, with 3-60-month follow-up. Obes Surg. 2000;10:233-9.

2. Haddad A, Tapazoglou N, Singh K, Averbach A. Role of intraoperative esophagogastroenteroscopy in minimizing gastrojejunostomy-related morbidity: experience with 2,311 laparoscopic gastric bypasses with linear stapler anastomosis. Obes Surg. 2012;22:1928-33.
3. Al Hadad M, Dehni N, Elamin D, Ibrahim M, Ghabra S, Nimeri A. Intraoperative endoscopy decreases postoperative complications in laparoscopic Roux-en-Y gastric bypass. Obes Surg. 2015;25(9):1711-5.

4. Champion JK, Hunt T, DeLisle N. Role of routine intraoperative endoscopy in laparoscopic bariatric surgery. Surg Endosc. 2002;16:1663-5.

5. Alasfar F, Chand B. Intraoperative endoscopy for laparoscopic RouX-en-Y gastric bypass: leak test and beyond. Surg Laparosc Endosc Percutan Tech. 2010;20(6):424- 7.

6. Sekhar N, Torquati A, Lutfi R, Richards WO. Endoscopic evaluation of the gastrojejunostomy in laparoscopic gastric bypass - A series of 340 patients without postoperative leak. Surg Endosc. 2006:20:199-201.

7. Carrodeguas L, Szomstein S, Zundel N, Lo Menzo E, Rosenthal R. Gastrojejunal anastomotic strictures following laparoscopic Roux-en-Y gastric bypass surgery: analysis of 1291 patients. Surg Obes Relat Dis. 2006;2(2):92-7.

8. Jamil LH, Krause KR, Chengelis DL, Jury RP, Jackson CM, Cannon ME, et al. Endoscopic management of early upper gastrointestinal hemorrhage following laparoscopic Roux-en-Y gastric bypass. Am J Gastroenterol. 2008;103:86-91.

9. Clayton RD, Carucci LR. Imaging following bariatric surgery: Roux-en-Y gastric bypass, laparoscopic adjustable gastric banding and sleeve gastrectomy. Br J Radiol. 2018;91(1089):20180031.

10. Mathew A, Veliuona MA, DePalma FJ, Cooney RN. Gastrojejunal stricture after gastric bypass and efficacy of endoscopic intervention. Dig Dis Sci. 2009;54:1971-8.

11. Schulman AR, Thompson CC. Complications of bariatric surgery: What you can expect to see in your GI practice. Am J Gastroenterol. 2017;112:1640-55.

12. Valenzuela-Salazar C, Rojano-Rodríguez ME, Romero-Loera S, Trejo-Ávila ME, Bañuelos-Mancilla J, Delano-Alonso R, et al. Intraoperative endoscopy prevents technical defect related leaks in laparoscopic Rouxen-Y gastric bypass: a randomized control trial. Int J Surg. 2017;50:17-21 